\title{
Ambivalence sémantique des noms de communication langagière du français
}

\author{
Jasmina Milićević \\ Dalhousie University \\ imilicev@dal.ca \\ Alain Polguère \\ Nancy-Université \& ATILF CNRS \\ alain.polguere@univ-nancy2.fr
}

\begin{abstract}
Nous nous intéressons dans cette étude aux noms de communication langagière du français, tels que REPRIMANDE, CRITIQUE, DEMANDE, TEMOIGNAGE, etc. Un trait saillant de nombre de noms de ce type est ce que nous appelons leur ambivalence sémantique: la même lexie (= unité lexicale) peut dénoter une communication langagière - donc un acte, un fait d'un type particulier - ou l'énoncé correspondant - donc une "entité linguistique» associée au fait en question. La description lexicographique des noms de communication langagière doit rendre compte de cette ambivalence sémantique, qui se reflète dans la structure de leur définition, dans les connexions paradigmatiques qu'ils entretiennent avec d'autres lexies et dans leur combinatoire. Nous esquissons une méthodologie pour le traitement lexicographique cohérent et uniforme des noms de communication langagière du français, méthodologie que nous croyons transférable à d'autres champs sémantiques et à des langues autres que le français. Notre présentation s'effectue en trois étapes : caractérisation de l'ambivalence sémantique, présentation des notions théoriques et descriptives sur lesquelles nous nous appuyons et, finalement, perspective lexicographique sur la question menant à des propositions pour un traitement lexicographique rigoureux de l'ambivalence sémantique.
\end{abstract}

\section{Caractérisation de l'ambivalence sémantique}

Avant toute chose, mentionnons que notre cadre théorique et descriptif est celui de la Lexicologie Explicative et Combinatoire (Mel'čuk et al., 1995). Bien que nous introduisions plus bas (section 2) plusieurs notions fondamentales appartenant à notre cadre de référence, il nous semble important de préciser dès maintenant deux éléments centraux de notre terminologie : lexie et vocable. Nous entendons par lexie, ou unité lexicale, l'entité lexicale qui est l'unité de description lexicologique/lexicographique, identifiée par un sens donné. La lexie correspond donc à ce qu'on appelle word-sense en anglais, étant entendu que le sens n'est que le paramètre principal de caractérisation de la lexie, paramètre qui est indissociable de deux autres paramètres : la forme (mots-formes ou syntagmes exprimant le sens lexical) et la combinatoire grammaticale et lexicale. La lexie peut, formellement, être un lexème (lexie monolexémique) ou une locution (lexie-syntagme).

Le vocable, quant à lui, est un regroupement de lexies partageant les mêmes signifiants et présentant une intersection de sens significative ; il correspond approximativement à une entrée dans la nomenclature d'un dictionnaire. Ainsi, selon notre terminologie, seules les lexies (et non les vocables) ont un sens propre, et la notion d'ambivalence sémantique relève d'une propriété des lexies, à distinguer clairement de la polysémie des vocables : propriété, pour les vocables, de regrouper plus d'une lexie. Pour une présentation des notions de lexie et vocable, voir notamment Polguère (2008).

Nous allons, dans cette première section, cerner la notion d'ambivalence sémantique des lexies. Elle sera tout d'abord introduite de façon très générale, à partir de l'analyse de plusieurs lexies du français. Nous examinerons ensuite le problème de la discrimination entre ambivalence sémantique (d'une lexie) et 
polysémie (d'un vocable), puis celui de la discrimination entre ambivalence sémantique et vague, pour finalement introduire le thème spécifique de cet article: l'ambivalence sémantique des noms de communication langagière du français.

\subsection{Lexies sémantiquement ambivalentes : caractérisation approximative}

Les lexies des langues se laissent normalement décrire, au niveau sémantique, au moyen des définitions que nous appelons analytiques (Polguère, 2008: 182-183). De telles définitions sont une décomposition paraphrastique du sens lexical structurée en deux composantes majeures :

- une composante centrale - aussi appelée genre prochain-, qui est la paraphrase minimale de la lexie et fonctionne comme pivot sémantique de sa définition ;

- une ou plusieurs composantes périphériques - aussi appelées différences spécifiques -, qui complètent la caractérisation apportée par la composante centrale en particularisant la lexie définie relativement à ses co-hyponymes et, plus généralement, en complétant la spécification de sa combinatoire sémantique.

Par exemple, la définition suivante du Trésor de la Langue Française informatisé (Dendien \& Pierrel, 2003), dorénavant TLFi, possède une structure de définition analytique :

AVION (acception A) : Appareil composante centrale plus lourd que l'air composante périphérique 1 pouvant voler grâce à différents procédés composante périphérique 2 et utilisé à des fins diverses composante périphérique 3 .

Certaines lexies ne se plient pas à ce schéma simple de définition, car elles présentent une ambivalence sémantique : elles possèdent au moins deux composantes centrales distinctes. Cette particularité sémantique se retrouve souvent explicitement encodée dans la structure des définitions des dictionnaires de langue par le recours à la conjonction de coordination ou (ou un outil linguistique équivalent); par exemple, toujours dans le TLFi :

INSULTE (acception B.1) : Paroles composante centrale 1 ou attitude composante centrale 2 (interprétables comme) portant atteinte à l'honneur ou à la dignité de quelqu'un (marquant de l'irrespect, du mépris envers quelque chose).

[Ex. 1 (paroles) : Tiens, tu mourras sans confession, vilain damné, qui n'a pas communié cette année. Cette insulte irrita le Chouan au point de le faire pâlir. (BALZAC, Chouans, 1829, p. 49);

Ex. 2 (attitude) : Ce geste lui apparut comme une insulte à l'amitié. (RADIGUET, Bal, 1923, p. 113)]

Note : Dans la mesure où il est difficile d'admettre qu'un geste est une attitude (cf. l'exemple 2), la seconde composante centrale de la définition ci-dessus devrait être élargie de façon à inclure aussi les actes, au sens large, et les comportements.

COCHONNERIE (acception B.2) : Action composante centrale 1 ou parole composante centrale 2 contraire à la décence, à l'honnêteté ou à la loyauté.

[Ex. 1 (action) : Nous parlons des dernières cochonneries politiques. (VALÉRY, Lettres à quelques-uns, 1945, p. 71) ;

Ex. 2 (parole) : Il lui disait des grosses cochonneries, des mots d'ordure. (ARAGON, Les Beaux-quartiers, 1936, p. 102)]

Parfois, la structure de la définition ne reflète pas explicitement la nature ambivalente de la lexie définie. Par exemple, il nous semble raisonnable de considérer XENOPHOBIE [On remarque plusieurs signes clairs d'une montée de la xénophobie à travers l'Europe.] comme ambivalente entre un sentiment négatif particulier et une attitude (liée à ce sentiment négatif). Pourtant, le TLFi évite l'emploi de deux composantes centrales coordonnées dans la définition de cette lexie, en subordonnant une des composantes à l'autre :

Hostilité $\Rightarrow$ sentiment négatif $_{\text {manifestée }}^{\Rightarrow \text { attitude }}$ à l'égard des étrangers, de ce qui est étranger. 
Une autre stratégie que les dictionnaires de langue utilisent pour traiter les lexies ambivalentes consiste à en donner une définition en deux blocs majeurs séparés par un point-virgule. Ainsi, la définition d'ADORATION [Le roi Hammurabi est ici représenté en adoration devant le Dieu Shamash.], qui selon nous est ambivalente entre une disposition psychique et la manifestation d'une croyance religieuse, se présente dans le TLFi comme suit :

Action d'adorer; attitude adoratrice.

Une lexie peut être sémantiquement ambivalente tout en ayant une définition ne possédant qu'une composante centrale - dans le cas où la composante centrale est elle-même ambivalente. Voici un tel cas repéré dans le TLFi :

MENSONGE (acception A) : Affirmation composante centrale contraire à la vérité composante périphérique 1 faite dans l'intention de tromper composante périphérique 2 .

[Ex. Francillon se penchait sur le livre avec une application pleine de défiance, comme s'il se fût proposé de déceler les mensonges de l'auteur. (SARTRE, Mort dans l'âme, 1949, p. 54)]

Il nous semble intuitivement clair qu'un mensonge peut désigner un acte (fait de mentir) ou l'énoncé correspondant (ce qu'on dit lorsqu'on ment). Un examen superficiel de quelques contextes d'emploi de MENSONGE permet de corroborer cette intuition; par exemple, dans les deux phrases suivantes relevées sur Internet :

- Ainsi Kelso devient-il l'ami intime de Williams, et par voie de conséquence le seul témoin du mensonge qui permettra à l'accusé d'être acquitté. $\Rightarrow$ mensonge acte d'énonciation

- Cette histoire est un mensonge. $\Rightarrow$ mensonge énoncé

Il s'agit là d'une ambivalence sémantique implicite, héritée par MENSONGE de sa composante centrale, 'affirmation', clairement explicitée dans le TLFi (cf. les deux composantes de la définition ci-dessous, séparées par un point-virgule) :

AFFIRMATION (acception B) : Action d'énoncer un jugement de valeur ou d'existence, d'authenticité ; le jugement énoncé qui en résulte.

Comme autre cas d'ambivalence, citons celui de LIVRE - objet physique support d'un contenu informationnel ou le contenu informationnel en question -, souvent mentionné dans les études de sémantique lexicale en regard de ce type de problème ; par exemple, dans le dictionnaire Lexis (Lexis, 1992) :

LIVRE (vocable 3, acception 1) : Volume imprimé d'une certaine étendue, considéré du point de vue de l'objet matériel ou celui du contenu.

[Ex. 1 (objet) : Livre relié, doré sur tranches ; Ex. 2 (contenu) : Il pensa aussi à un écrivain dont il aimait les livres. (Bastide)]

La définition ci-dessus semble à première vue reléguer la disjonction sémantique dans les composantes périphériques (considéré...). Cependant, il s'agit en fait d'expliciter les deux interprétations du terme volume, choisi comme composante centrale: volume en tant que dénotant un objet physique ou un contenu informationnel. Les deux exemples proposés par le Lexis (le premier exemple construit et la citation) servent, comme dans le cas de INSULTE dans le TLFi, à « épauler » la définition dans la spécification de l'ambivalence sémantique de LIVRE. On pourrait reformuler la définition du Lexis de la façon suivante, équivalente du point de vue du contenu :

Volume imprimé d'une certaine étendue ou son contenu.

Le TLFi et le Petit Robert (Petit Robert, 2010), qui ont une approche beaucoup plus diachronique de la structure de leurs entrées que le Lexis, distinguent pour LIVRE deux familles d'acceptions, selon que l'on 
considère l'objet physique ou le contenu informationnel. Cette observation nous amène tout naturellement au problème du rapport entre ambivalence sémantique et polysémie.

\subsection{Ambivalence sémantique vs polysémie}

Si l'on adopte une perspective lexicographique sur le traitement de l'ambivalence sémantique - perspective qui est celle qui nous intéresse en tout premier lieu ici-, on voit qu'il est nécessaire de faire un choix entre deux descriptions possibles :

1. on considère, comme le fait le Lexis pour LIVRE, une lexie unique, sémantiquement ambivalente - c'està-dire, une acception unique du vocable LIVRE dont la définition contient une disjonction (normalement, dans sa composante centrale) ;

2. on considère, comme le font le TLFi et le Petit Robert pour LIVRE, qu'il n'y a pas d'ambivalence sémantique, car l'on est en présence de deux lexies-copolysèmes - c'est-à-dire, deux acceptions distinctes du vocable LIVRE.

Selon notre approche théorique et descriptive, qui est, tel qu'indiqué plus haut, celle de la Lexicologie Explicative et Combinatoire, le choix entre les deux options doit s'opérer sur la base des critères suivants, qui identifient les cas correspondant à l'option 1 - l'identification d'une seule lexie, sémantiquement ambivalente.

Deux contenus sémantiques potentiellement associables à deux lexies distinctes doivent être associés à une seule et unique lexie si l'on constate les faits suivants :

- Combinatoire sémantique compatible. Il est possible d'employer, dans un même énoncé, une occurrence unique de la lexie en question en cooccurrence avec deux segments linguistiques compatibles chacun avec un des contenus sémantiques. Il s'agit ici du critère de cooccurrence compatible (aussi appelé critère de Green-Apresjan). Par exemple, l'énoncé suivant montre que insulte peut s'utiliser une unique fois dans un énoncé pour référer simultanément à des paroles et à des comportements/attitudes et que, donc, le TLFi a probablement raison de postuler une seule lexie (sémantiquement ambivalente) : Je ne supporte plus les insultes qu'il me fait subir: que ce soit ses paroles vulgaires ou ses gestes méprisants. Par contraste, les deux dénotations possibles de copie - acte de copier vs document résultant ne peuvent pas être portées simultanément dans un énoncé par une même occurrence de copie, sous peine de produire un zeugme - signalé ici par le symbole d'incohérence sémantique «" $":$ : Bien que la copie $d u$ bouquin ait duré toute la matinée, $j$ 'ai dû la déchirer ensuite. Cela nous conduit à postuler que l'on a ici deux lexies distinctes du vocable COPIE. Pour tester la cooccurrence compatible, on utilise aussi souvent le test de lectures croisées (dont une des variantes est connue en anglais sous le nom de do so test). Selon ce test, les deux dénotations possibles de insulte peuvent être regroupées sous une seule lexie : Ses paroles vulgaires furent une insulte; ses gestes méprisants aussi <tout comme ses gestes méprisants>.

- Liens paradigmatiques identiques. Les lexies liées paradigmatiquement à la lexie décrite, notamment ses dérivés sémantiques (Mel'čuk \& Polguère, 2007 : 18-19), ne se subdivisent pas en deux blocs, selon qu'ils sont associables à l'une ou l'autre des interprétations possibles. Par exemple, les dérivés sémantiques suivants de insulte conviennent aussi bien pour une insulte verbale que pour insulte-attitude : insulter, insultant, sous [ART insulte] (cf. Il bondit sous l'insulte). Si l'on constate le phénomène inverse plusieurs blocs de liens paradigmatiques, chacun associable à l'un des contenus sémantiques considérés -, on est en présence de plusieurs lexies distinctes satisfaisant ce que l'on appelle le critère de dérivation différentielle (Mel'čuk et al., $1995: 68$ ).

- Liens syntagmatiques identiques. On ne constate pas une séparation entre deux familles de collocatifs, selon qu'ils sont compatibles avec l'un ou l'autre des contenus sémantiques. Par exemple, insulte cruelle, subir des insultes, répondre à une insulte, etc. sont toutes des collocations compatibles avec insulte en tant 
que paroles et en tant que comportement/attitude. Bien entendu, il peut arriver que certains collocatifs soient plus directement compatibles avec l'une ou l'autre des lectures, mais ce qui compte, c'est qu'il n'y ait pas de généralisation de ce phénomène dans la combinatoire lexicale de la lexie décrite. Dans le cas contraire, nous sommes en présence de deux lexies distinctes, qui satisfont ce que l'on appelle le critère de cooccurrence différentielle (Mel'čuk et al., $1995: 66$ ).

Pour une discussion des critères de délimitation des lexies dans le cadre de la Lexicologie Explicative et Combinatoire, voir (Mel'čuk et al. (1995 : 57-69) et Mel'čuk (2006 : section 3.2). Kleiber (2008) offre un tour d'horizon de critères et tests d'identification de la polysémie, incluant ceux-ci et d'autres, comme, par exemple, la non-unifiabilité vériconditionnelle, qui se manifeste comme la possibilité de négation indépendante. Ainsi, il est possible de répondre par Non! à la question As-tu une souris ? si celle-ci porte sur une souris-animal, alors qu'on possède par ailleurs une souris d'ordinateur. De façon plus générale, on remarquera que le problème de la spécification de l'ambivalence sémantique et de la distinction entre ambivalence et polysémie est en lien direct avec les notions établies par Cruse (1995) de sub-sense et facet of a lexical unit. Ce problème est aussi étudié dans l'approche du lexique génératif (Pustejovsky, 1995, 1998) sous le nom de polysémie logique, et modélisé grâce à l'outil formel des types pointés (dotted types) - pour une brève synthèse sur la notion de polysémie logique, voir Jacquey (2006a). Pour ce qui est des travaux traitant de cette problématique sur la base du lexique français, on peut mentionner notamment Godard \& Jayez (1996), où il est question de noms multitypés, Jacquey (2006b), qui décrit en tant que polysémie logique l'ambivalence du type 'action de créer qqch.' 'objet créé', et Kleiber (1999), qui traite de l'ambivalence comme un cas particulier de métonymie. Néanmoins, tous ces travaux ont une orientation nettement de sémantique «pure », appliquée à l'analyse des énoncés, alors que nous nous intéressons, comme nous l'avons dit, avant tout aux aspects lexicographiques de la modélisation de la connaissance lexicale et du phénomène d'ambivalence. Le problème spécifique de la résolution d'ambiguïté, notamment, ne nous concernera aucunement puisque nous travaillons, en quelque sorte, en amont de ce dernier : au niveau des connaissances de sémantique lexicale utilisées par le locuteur lorsqu'il construit son énoncé.

Formellement, nous considérons que le sens des lexies ambivalentes est organisé autour de plusieurs composantes centrales réunies par une disjonction. Plus précisément, puisque ces lexies satisfont le critère de combinatoire sémantique compatible, nous considérons que les différentes composantes centrales sont réunies par une disjonction inclusive « et/ou» et non par une disjonction exclusive « ou»: «P et/ou $\mathrm{Q}$ » est toujours vrai, sauf quand « $\mathrm{P} »$ et « $\mathrm{Q} »$ sont simultanément faux, alors que « $\mathrm{P}$ ou $\mathrm{Q} »$ est toujours faux, sauf quand « $\mathrm{P}$ » et « $\mathrm{Q} »$ sont simultanément vrais. Ainsi, la définition que nous avons proposée plus haut pour LIVRE devrait être reformulée de la façon suivante :

Volume imprimé d'une certaine étendue et/ou son contenu.

$\mathrm{Du}$ point de vue du locuteur, la différence entre une disjonction inclusive $v s$ exclusive des composantes centrales est fondamentale. La définition ci-dessus nous indique que le locuteur peut utiliser livre dans un énoncé donné soit pour dénoter une entité physique, soit pour dénoter un contenu informationnel, soit pour dénoter simultanément les deux.

On remarquera d'ailleurs qu'il arrive aux dictionnaires de langue de faire un usage explicite (mais non systématique) de la disjonction inclusive pour connecter les composantes centrales d'une lexie décrite comme étant ambivalente ; par exemple, dans le TLFi :

AVANCER (acception II.B.2) : Aller trop vite et/ou indiquer une heure en avance sur l'heure, sur le temps réel.

[Ex. : Elle [Marie-Antoinette] recommença la même erreur. Elle s'aperçut ensuite qu'elle s'était trop prévenue et que Barnave revenait à eux. C'est ainsi que l'horloge des Tuileries retardait toujours : le cadran de l'Hôtel de Ville avançait. (SAINTE-BEUVE, Nouveaux lundis, t. 8, 1863-69, p. 373.)] 
Les lexies sémantiquement ambivalentes typiques ont donc leur sens structuré autour d'une disjonction inclusive de composantes centrales. Il est cependant aussi possible de considérer un cas particulier de lexies ambivalentes, sémantiquement construites autour d'une disjonction exclusive. En effet, même lorsque le nonrespect du critère de combinatoire sémantique compatible devrait nous pousser à distinguer deux lexies, nous pouvons être amenés à considérer une et une seule lexie lorsque les deux conditions suivantes sont remplies :

1. les deux éléments de contenu considérés peuvent être reliés par une règle de dérivation sémantique très régulière dans la langue ;

2. il est possible de spécifier dans la règle de dérivation sémantique en question toutes les caractéristiques d'expression du sens dérivé.

Prenons ainsi le cas des deux contenus sémantiques 'animal' et 'espèce animale correspondante', que l'on trouve instanciés, respectivement, dans Je vais promener le chien $\sim$ Le chien est le meilleur ami de l'homme. Bien que très régulière, la dérivation sémantique nom d'animal $\rightarrow$ nom d'espèce animale correspondante est d'application arbitraire en français puisque, notamment, c'est parfois le nom de l'animal mâle qui est la source de la dérivation (cf. chien) et parfois le nom de l'animal femelle (cf.poule). Il faut donc que la description du vocable correspondant prenne en charge cette apparente polysémie. Cependant, il est contreintuitif de multiplier des descriptions de lexies dénotant des espèces animales, en tant que co-polysèmes des lexies dénotant les animaux eux-mêmes, dans la mesure où ces lexies dérivées n'ont généralement pas de propriétés propres qui ne pourraient être associées à la règle générale de dérivation. Ainsi, le fait que chienespèce s'emploie avec un défini générique découle entièrement de sa nature sémantique de 'nom d'espèce'. On pourra donc vouloir considérer que l'on a ici une seule et unique lexie présentant un cas extrême d'ambivalence sémantique reposant sur une disjonction exclusive de composantes centrales. (Il est bien entendu impossible d'utiliser une seule occurrence de cette lexie pour dénoter simultanément un animal spécifique et l'espèce correspondante.) Bien que périphérique, ce cas particulier d'ambivalence sémantique doit être pris en considération puisqu'il implique la présence de définitions à composantes centrales multiples, reposant cette fois-ci sur une disjonction exclusive.

Mais ce n'est pas tout. Il peut aussi arriver que la dérivation sémantique soit non plus simplement régulière, mais aussi systématique : c'est-à-dire que, pour tout sens de la langue d'un type donné, un autre sens puisse être systématiquement dérivé, la règle ne souffrant aucune exception. Tel est le cas des noms du type MEDECIN, qui dénotent un individu exerçant une activité professionnelle donnée - Il a vu un médecin -, et la profession correspondante - Médecin est une profession encore très respectée. Si nous voulions construire une définition lexicographique d'une unique lexie MEDECIN en indiquant explicitement les deux dénotations possibles de la lexie en question, il nous faudrait bien entendu avoir recours à une disjonction exclusive, par exemple :

Personne habilitée à exercer la médecine ou profession correspondante.

Cependant, la dérivation en question étant systématique, une telle définition semble inutile, et même un peu ridicule. Plutôt que de spécifier dans le dictionnaire que MEDECIN peut avoir deux dénotations, il est plus économique et, surtout, plus exact d'indiquer dans une règle sémantique générale du français que tous les noms de cette langue dénotant un individu exerçant une certaine profession peuvent aussi être utilisés pour dénoter la profession en question. On a donc avec MEDECIN une lexie «virtuellement» ambivalente, dont l'ambivalence sémantique n'est pas prise en compte par le dictionnaire - le modèle du lexique -, mais par une règle sémantique générale - voir à ce propos la coercition de type dans l'approche du lexique génératif et la métonymie intégrée de G. Kleiber.

Les deux cas de disjonction exclusive nous venons d'examiner relèvent de la distinction connue sous le nom de polysémie régulière vs polysémie systématique - voir Apresjan (1974) et Barque (2008). Il convient cependant de relever que, dans la mesure où aucun de ces deux cas de figure ne nous amène à postuler, au 
niveau du dictionnaire, deux lexies distinctes, il ne s'agit pas de cas de polysémie véritable (présence dans le dictionnaire de plusieurs acceptions) des vocables impliqués. Pour le même type de raison, nous écartons l'usage de termes comme polysémie logique, mentionné plus haut, pour dénoter le phénomène d'ambivalence sémantique reposant sur une disjonction inclusive de composantes centrales, puisqu'il ne s'agit précisément pas de polysémie (multiplicité des acceptions). La «vision polysémique » de l'ambivalence sémantique est bien souvent motivée par une approche de décodage des énoncés (perspective du destinataire) plutôt que par une approche d'encodage de ceux-ci (perspective du locuteur). Dans la première approche, on est confronté au problème de la résolution d'ambiguïtés et toute présence d'ambiguïté est automatiquement associée à un cas de polysémie. Or l'ambiguïté (propriété des énoncés) n'est que partiellement liée à la polysémie (propriété des vocables), qui n'en est qu'une des causes possibles, et les deux notions ne doivent aucunement être confondues.

Comme on le voit, les rapports entre ambivalence sémantique et polysémie sont complexes et nous proposons de les aborder sous un angle véritablement lexicographique : c'est la logique de la méthode lexicographique et les principes qu'elle met en jeu qui nous pousse vers tel ou tel type de modélisation.

\subsection{Ambivalence sémantique vs vague}

Nous venons de voir que l'ambivalence sémantique des lexies, dans le cas le plus typique, repose sur une disjonction inclusive de composantes centrales de leur définition. Or, une telle disjonction peut également être présente dans la définition de lexies sémantiquement vagues. Il convient donc de tracer la frontière entre les sens ambivalents et les sens vagues.

L'expression sens vague ne semble pas avoir de statut terminologique précis, même s'il est commun d'insister, notamment, sur la distinction entre les deux notions de vague et ambigu. Elle est utilisée en tant que synonyme de sens général ou sens sous-spécifié et apparaît plutôt dans un contexte relationnel : un sens est considéré comme étant vague par rapport à un autre. Ainsi, on dit que enfant est vague par rapport à garçon et fille, que se déplacer est vague par rapport à marcher, courir, voler, etc. ou que le nom russe ruká, qui recouvre l'espace conceptuel partagé en français par main et bras, est vague par rapport à ces derniers. Il arrive cependant que l'on parle de vague tout court, de façon absolue ; par exemple, on dit que le nom baiser est vague, puisqu'il il peut dénoter indifféremment un baiser à caractère sexuel (baiser fougueux) ou non (baiser d'adieu). Ou encore, l'adjectif chauve serait vague puisque le locuteur, tout en connaissant son sens 'dégarni de cheveux' (acception A du TLFi) -, peut trouver difficile de déterminer s'il s'applique ou non dans une situation donnée, à cause du caractère gradable de la propriété correspondante. (On peut être totalement, quasiment ou presque chauve.)

Il existe un type de vague reposant sur une disjonction de composantes centrales dans la définition des lexies correspondantes. Il est fréquemment illustré à partir de lexies désignant des liens de parenté ; ainsi, on trouve dans le Petit Robert:

TANTE (acception 1) : Sœur du père ou de la mère, et par ext. Femme de l'oncle.

Cette définition peut être reformulée ainsi, de façon plus explicite :

TANTE (acception 1) : Sœur du père et/ou sœur de la mère et/ou femme de l'oncle.

On remarquera que nous sommes ici en présence d'une série de disjonctions inclusives. En effet, les lexies vagues - tout comme les lexies sémantiquement ambivalentes typiques - répondent au critère de cooccurrence compatible, comme l'illustre la phrase suivante : Mes trois tantes étaient là : Luce, la sœur de ma mère, Yvonne, la sœur de mon père, ainsi que Constance, la femme de mon oncle Gérard. Plus que cela, les lexies vagues se comportent exactement comme les lexies ambivalentes vis-à-vis de tous les autres critères de délimitation de lexies que nous avons cités précédemment; pour une discussion, voir Kleiber (1998). 
Pourquoi ne voulons-nous pas assimiler de tels cas de vague à de l'ambivalence sémantique ? Parce que le rapport entre les éléments disjonctifs est, dans les deux cas d'ambivalence et de vague, de nature bien différente. Les composantes disjonctives dans le sens des lexies ambivalentes sont conceptuellement distinctes, leur « distance conceptuelle » pouvant aller jusqu'à la distance entre faits et entités (voir plus bas, section 2.1). Ce n'est pas du tout le cas avec les lexies vagues : les différentes composantes de leur définition dénotent des «variantes » d'une même entité ou d'un même fait général. Cette particularité du vague est d'ailleurs très clairement mise en évidence par Kleiber (2008: 91) lorsqu'il parle d'unifiabilité catégorielle dans le cas des lexies vagues.

Il est donc légitime de considérer que les lexies vagues ne sont pas ambivalentes, mais bien « monovalentes », et cela, que leur nature vague soit modélisée ou non au moyen d'une disjonction inclusive de composantes centrales. On verra plus bas, lors de l'examen leur étiquetage sémantique, que tous les noms de communication langagière ambivalents que nous considérons ici se distinguent bien de lexies vagues par l'écart conceptuel existant entre les deux types de dénotations qu'ils peuvent avoir.

\subsection{Ambivalence sémantique des noms de communication langagière (NCL) du français}

Nous nous intéresserons maintenant à une classe sémantique particulière de noms communs français, ceux qui ont dans leur définition, en tant que composante centrale — ou en tant qu'une des composantes centrales, dans un cas d'ambivalence sémantique - le sémantème 'communication langagière' (ou un sémantème plus spécifique).

Nous appellerons noms de communication langagière - désormais, NCL - les noms de ce type. Voici trois exemples de NCL français :

1. COMPLIMENT\#a [Il débita à l'assistance émue un compliment bien tourné.],

2. QUESTION\#1a [Elle répondra à toutes les questions qu'on lui a posées.],

3. TEMOIGNAGE\#I.1 [Selon le témoignage d'un passant, une altercation aurait éclaté entre les deux hommes peu avant la fermeture du bar.].

Remarque : Nous utilisons ici la numérotation des lexies telle qu'elle apparait dans la base lexicale DiCo (Polguère, 2000), dans sa version actuelle. Pour éviter toute confusion, les numérotations lexicographiques empruntées au DiCo apparaissent dans cet article en police non proportionnelle, précédées du symbole \#.

À notre connaissance, les NCL du français n'ont pas été étudiés en tant que tels. Même pour l'anglais, nous ne connaissons pas d'étude poussée des noms de communication, alors qu'il existe un foisonnement de recherches sur les verbes correspondants - par exemple, le fameux Dictionary of English speech act verbs d'Anna Wierzbicka (Wierzbicka, 1987). Les NCL du français, bien qu'ils ne semblent pas avoir retenu l'attention des lexicologues, sont particulièrement intéressants en ce qu'ils tendent à manifester une forme d'ambivalence sémantique, qui devrait être prise en compte dans leur définition lexicographique. De plus, cette ambivalence n'est souvent pas aisée à identifier et à modéliser de façon rigoureuse. Examinons deux cas illustrant ce problème.

1. QUESTION\#1a [Sa question tient en deux mots : «Pour quoi ?».]

Cette lexie, comme le montre la phrase exemple, dénote un type particulier d'énoncé. Cependant, elle peut aussi être utilisée - et il ne s'agit pas là de polysémie ! - pour dénoter un acte d'énonciation, comme l'illustre l'exemple suivant relevé sur un site Internet journalistique : Regardez bien les tics présidentiels 
durant $_{\Rightarrow \text { fait }}$ la question. Insistons sur le fait qu'il s'agit d'une seule lexie, car elle satisfait aux critères donnés dans la section 1.2 ci-dessus; notamment, on peut utiliser de façon ambivalente une unique occurrence de question \#1 a dans la phrase : Regardez bien les tics présidentiels durant $\Rightarrow$ fait la question, qui apparaît dans sa traduction anglaise en bas de l'écran $\Rightarrow$ énoncé.

2. REPRIMANDE [Alors, elle fugue, revient à Colmar, prête à affronter les pires réprimandes.]

Cette lexie peut clairement être considérée comme une nominalisation - en termes de fonctions lexicales (Mel'čuk, 1996), un $\mathbf{S}_{\mathbf{0}}-\mathrm{du}$ verbe REPRIMANDER : réprimande de $X=$ le fait que $X$ réprimande/a réprimandé/réprimandera. Elle dénote donc un fait, plus précisément une communication langagière. Cependant, elle peut tout autant dénoter - et, là encore, il ne s'agit pas de polysémie - un énoncé ou un texte (= ensemble d'énoncés) par lequel on réprimande ; par exemple : réprimande écrite sur le carnet de correspondance (trouvé sur un site Internet scolaire officiel décrivant une «échelle de punitions scolaires $\gg)$.

Comme on le voit, nous avons présenté les deux cas ci-dessus de façon inverse l'un vis-à-vis de l'autre : QUESTION\#1a avant tout comme un énoncé et, de façon secondaire, une communication langagière ; REPRIMANDE avant tout comme une communication langagière et, de façon secondaire, un énoncé/texte. Cela est en correspondance avec une perception intuitive qu'auront beaucoup de francophones d'une sorte d'asymétrie de l'ambivalence, même si cette dernière est assez difficile à démontrer et peut par ailleurs sembler contestable à certains. Il nous semble que c'est là un des autres aspects remarquables du sémantisme des NCL, qui rend leur modélisation particulièrement ardue.

Ces difficultés de modélisation sont apparentes dans les dictionnaires de langue et un examen superficiel du traitement lexicographique des NCL permet de relever de nombreuses hésitations et incohérences. Considérons, à titre d'illustration, un seul exemple, celui du traitement de l'acception de base de QUESTION dans le Petit Robert et sa contrepartie dans le TLFi.

[Petit Robert] QUESTION (acception I.1) : Demande qu'on adresse à qqn en vue d'apprendre qqch. de lui.

Il n'y a rien dans la forme de la définition du Petit Robert qui laisse présager une ambivalence sémantique. Cette définition privilégie nettement le sens 'acte' de QUESTION I.1 ; d'ailleurs, l'examen de la définition de DEMANDE I.1 (la composante centrale de QUESTION I.1) dans le même dictionnaire confirme cette interprétation :

[Petit Robert] DEMANDE (acception I.1) : Action de demander, de faire connaître à qqn ce qu'on désire obtenir de lui.

Examinons maintenant la description de QUESTION ${ }^{\mathbf{1}} \mathbf{1}$ dans le TLFi - l'acception du vocable QUESTION qui correspond à QUESTION I.1 du Petit Robert et à QUESTION\#1a du DiCo :

[TLFi] QUESTION (vocable 1, acception I) : Demande adressée en général oralement à quelqu'un, soit sous la forme d'une phrase incomplète qui appelle un complément, une confirmation, ou une dénégation, soit sous la forme d'une interrogation, pour en apprendre quelque chose, en obtenir une réponse, en connaître l'opinion; l'énoncé de cette demande.

On voit clairement ici une définition bicéphale, même si le TLFi ne fait pas usage d'une conjonction disjonctive ; la disjonction est en fait exprimée par le recours au point-virgule. La première « sous définition » est structurée autour de la composante centrale 'demande', qu'il faut ici interpréter, contrairement à ce qui a été fait pour la définition du Petit Robert, comme dénotant un segment linguistique. Notamment, on doit absolument donner une interprétation 'énoncé' à cette partie de la définition pour pouvoir expliquer en quoi elle se distingue de la seconde partie, clairement de type 'acte' : énoncé de cette demande. Rappelons la 
polysémie du terme énoncé: un énoncé [='segment linguistique'] vs l'énoncé de qqch. [='acte d'énonciation'].

Remarque : Un projet de balisage XML des définitions du TLFi identifiant les différentes composantes sémantiques de celles-ci, projet appelé Definiens, est en cours de réalisation (Barque \& Polguère, 2009 ; Nasr et al., 2010). Selon les conventions adoptées dans le cadre de Definiens, une définition telle que celle de QUESTION ${ }^{1} \mathbf{1}$ ci-dessus est balisée comme étant constituée de deux paraphrases distinctes (balise XML PARAPH). Certaines lexies sémantiquement ambivalentes sont cependant définies dans le TLFi par une paraphrase unique (donc, sans recours au point-virgule), l'ambivalence apparaissant comme une coordination syntaxique disjonctive (balise XML ET_OU) au sein de la composante centrale (balise XML CC). Tel est le cas, par exemple, des définitions de INSULTE B.1 et COCHONNERIE B.2 données plus haut, au début de la section 1.1.

Résumons les faits. Alors que le Petit Robert décrit l'acception de base du vocable QUESTION comme dénotant avant tout (uniquement?) un acte de communication, le TLFi la décrit comme sémantiquement ambivalente en ayant recours à une définition multiple (et non à une définition à composante centrale multiple). Il ne s'agit que d'un exemple particulier de divergence de description, mais une étude systématique de la description lexicographique des NCL du français montrera nombre de divergences de ce type, de même que des incohérences à l'intérieur d'un même dictionnaire. Cela démontre selon nous la réalité de la difficulté de définir les NCL du français et la nécessité de proposer un traitement uniforme et cohérent de leur sémantisme. Dans la suite de la présente étude, nous chercherons à apporter quelques lumières sur la question, sans toutefois prétendre la régler de façon définitive. Nous proposons notamment dans l'Annexe, en fin d'article, une définition plus adéquate de la lexie QUESTION I.1/QUESTION ${ }^{1}$ 1/QUESTION\#1a.

Nous en avons maintenant terminé avec ce tour d'horizon général de l'ambivalence sémantique. Avant de passer à la section suivante, qui présente brièvement les notions clefs que nous utiliserons dans notre description des NCL, il convient de faire deux remarques importantes.

Premièrement, tout ce que nous disons ici concerne les NCL du français uniquement, même s'il est probable que les phénomènes que nous examinons ont une contrepartie dans nombre d'autres langues. Nous ne sommes simplement pas en mesure, pour l'instant, de proposer une étude interlinguistique suffisamment étayée.

Deuxièmement, les NCL présentent d'autres types d'ambivalences sémantiques que celles que nous avons présentées. Par exemple, les NCL comme COCHONNERIE (vu auparavant), BETISE ou ANERIE sont ambivalents entre actes et paroles. Certains NCL peuvent aussi dénoter des communications non langagières (notamment, gestuelles), comme le montrent les deux illustrations suivantes :

- ENCOURAGEMENT : Durant la partie, le public doit s'abstenir de tout encouragement verbal ou gestuel.

- REPONSE : Ma réponse fut « Non », mais la sienne un hochement de tête affirmatif.

Cette ambivalence concerne aussi bien le NCL que le verbe correspondant (répondre positivement de la voix et d'un hochement de tête). 


\section{Notions descriptives et théoriques de base}

\section{1 Étiquettes sémantiques}

Nous adoptons ici l'approche lexicographique utilisée pour l'élaboration de la base lexicale DiCo (Polguère, 2000), approche qui met en jeu de façon essentielle les étiquettes sémantiques (Milićević, 1997 ; Polguère, 2003).

Une étiquette sémantique est soit un sémantème - qui s'exprime par une lexie de la langue (lexème ou locution) - soit une configuration de sémantèmes - qui s'exprime par un syntagme construit - qui apparaît en tant que composante centrale dans la définition d'un nombre significatif de lexies; par exemple, sentiment, événement, état, individu, dispositif, substance, domaine d'activité, communication langagière, etc. (Par convention, nous écrivons ici les noms d'étiquettes sémantiques en police non proportionnelle.) Certaines étiquettes pourront être beaucoup plus spécifiques que celles qui viennent d'être mentionnées, mais elles devront toujours rester « classifiantes », c'est-à-dire qu'elles devront toujours être la composante centrale de la définition d'au moins deux lexies de la langue.

Les étiquettes sémantiques sont organisées en une hiérarchie fondée sur la relation d'hyperonymie et structurée en premier lieu autour de l'ensemble des étiquettes nominales. Chaque étiquette nominale, cependant, peut se voir associer son corrélat dans une autre partie du discours (verbe, adjectif, adverbe et proposition), ce qui permet d'intégrer au sein d'une seule et unique hiérarchie l'ensemble des étiquettes sémantiques de la langue décrite.

Il est important de souligner que les étiquettes sémantiques, même si elles peuvent être ambiguës dans leur formulation, s'interprètent de façon univoque. Les lexèmes utilisés dans les noms d'étiquettes correspondent à une acception spécifique d'un vocable. Par exemple, parmi les trois acceptions du vocable EVENEMENT distinguées dans le TLFi, c'est à l'acception B-1 que correspond notre étiquette sémantique événement :

A vieilli 'fait auquel aboutit une situation' [L'évènement lui a donné raison.] ;

B.1 'tout ce qui se produit, tout fait qui s'insère dans la durée' [L'art photographique (...) consiste surtout à capter l'événement fugace, l'accident (...)] ;

B.2 'fait d'une importance notable pour un individu ou une communauté humaine' [Un bon livre est un événement.].

Aux fins de désambiguïsation, chaque étiquette sémantique que nous utilisons a été associée à une définition du TLFi et munie d'un numéro lexicographique distinctif. Cependant, pour alléger la description lexicographique, ces numéros distinctifs n'apparaissent pas dans les noms d'étiquettes.

Chaque étiquette sémantique identifie une classe de lexies dont elle est la composante centrale, sur la base de certaines propriétés de combinatoire. Comme les étiquettes sémantiques s'organisent en une hiérarchie, elles permettent de hiérarchiser de la même façon les classes de lexies qu'elles définissent et de déterminer ainsi une structuration de l'ensemble du lexique sur une base sémantique.

La hiérarchie des étiquettes sémantiques, développée de façon inductive lors de l'élaboration du DiCo, se subdivise en deux sous-hiérarchies principales à partir de l'étiquette mère qqch.: sous-hiérarchie de entité 'qqch. qui existe' et sous-hiérarchie de fait 'qqch. qui a/peut avoir lieu'. Alors qu'une lexie donnée n'est normalement associée qu'à l'une de ces deux sous-hiérarchies, nous verrons plus bas que les NCL ambivalents représentent une exception lexicale, puisqu'ils ont le potentiel de dénoter aussi bien des faits et des entités. 
Le sens de chaque lexie du DiCo est caractérisé au moyen d'une étiquette sémantique. Avec les lexies sémantiquement ambivalentes, trois cas de figure d'étiquetage peuvent se présenter. Ceux-ci reflètent les trois types d'ambivalence isolés plus haut, dans la section 1.2, en fonction (i) de la nature de la disjonction impliquée (inclusive ou exclusive) et (ii) du caractère simplement régulier - c'est-à-dire, descriptible au moyen d'une règle générale - ou systématique - c'est-à-dire, descriptible au moyen d'une règle générale d'application systématique - de l'ambivalence.

$1 \mathrm{Si}$ on est en présence d'une ambivalence typique, qui repose sur une disjonction inclusive de composantes centrales dans la définition, on choisira une étiquette explicitement bicéphale de type É1 et/ou É2 ; par exemple : disposition psychique et/ou manifestation d'une croyance religieuse pour ADORATION\#b [Il s'est agenouillé, en adoration devant Dieu.].

2 Si l'on est en présence d'une ambivalence reposant sur une disjonction exclusive de composantes centrales, dont on a des raisons de penser qu'elle est régulière mais non systématique, on maintient une structure d'étiquetage sémantique explicitement bicéphale de type É1 ou É2; par exemple, animal de compagnie ou espèce animale correspondante pour CHAT.

$3 \mathrm{Si}$, par contre, on est en présence d'une ambivalence par disjonction exclusive dont on a des raisons de penser qu'elle est régulière et systématique, on formulera l'étiquette comme un syntagme É1 $\rightarrow$ É2, où l'étiquette É1 est syntaxiquement dominante, ce qui lui confère en même temps une dominance communicative (Mel'čuk, 2001:30sqq); par exemple: individu qui pratique un métier pour AVOCAT\# $\#^{1} I$ [Ces jeunes gens avaient les moyens de se payer un bon avocat.]. Ici, on n'a donc pas de disjonction explicite dans l'étiquetage, mais l'ambivalence sera rendue par le fait que l'étiquette individu qui pratique un métier possède, dans la hiérarchie des étiquettes, deux étiquettes-mères individu et métier.

Remarquons que, jusqu'à présent, cette stratégie d'étiquetage n'a pas été appliquée de façon systématique dans le DiCo, notamment pour ce qui a trait à la distinction entre ambivalence par disjonction inclusive vs disjonction exclusive. Nous espérons justement que la présente étude permettra d'améliorer le système des étiquettes sémantique et son utilisation dans les bases lexicales du type DiCo.

Dorénavant, nous allons raisonner strictement en termes d'étiquettes sémantiques, sachant que, pour nous, le fait de dire qu'une lexie est étiquetée par l'étiquette É implique que la composante centrale de sa définition lexicographique doit être une expression linguistique (mono- ou multilexémique) formulée directement en termes de É. En effet, un de nos objectifs, dans le recours aux étiquettes sémantiques, est de normaliser l'écriture des définitions lexicographiques, comme nous le verrons dans la section 3.2.

La notion d'étiquette sémantique étant maintenant introduite, nous pouvons reformuler plus rigoureusement celle de NCL : nous dirons qu'un NCL est une lexie étiquetée soit par l'étiquette É1 ci-dessous - ou une de ses descendantes dans la hiérarchie - soit par une disjonction (inclusive ou exclusive) de É1 avec É2 ou É3 - ou une de leurs descendantes dans la hiérarchie :

1. É1: communication langagière;

2. É2 : énoncé, c'est-à-dire, « support linguistique » d'une communication langagière - dans le DiCo, cette étiquette correspond au lexème ENONCE\#1 [énoncé interrogatif; énoncé de cinq mots], distinct de ENONCE\#2 [faire l'énoncé du problème] ;

3. É3 : texte, c'est-à-dire, ensemble structuré d'énoncés.

Nous avons préféré l'étiquette (acte de) communication langagière à énonciation pour dénoter l'acte linguistique, car la seconde correspond à un prédicat sémantique biactanciel ('énonciation par X 
de $\left.Y^{\prime}\right)$ alors que nous désirons une étiquette triactancielle, rendant bien compte de la «transaction» linguistique : 'communication langagière par $\mathrm{X}$ de $\mathrm{Y}$ à $\mathrm{Z}$ '. Notons que l'étiquette verbale correspondant à communication langagière est dire (oralement ou par écrit).

\subsection{Degré de prédicativité des sens lexicaux}

Un paramètre qui s'avère pertinent pour notre description des NCL est leur nature prédicative particulière. Par nature prédicative des sens lexicaux, nous faisons référence à leur appartenance à l'une des trois classes sémantiques suivantes (Mel'čuk \& Polguère, 2008).

- Les prédicats sémantiques sont des sens qui se caractérisent par deux propriétés fondamentales. La première propriété est que ce sont des sens «liants», c'est-à-dire qu'ils appellent auprès d'eux l'expression d'autres sens - les arguments du prédicat ou, dans notre terminologie, les actants sémantiques du sémantème correspondant. La définition d'une lexie dont le sens est un sémantème prédicatif spécifie au moyen des variables $X, Y$, etc. les positions actancielles anticipées par le sémantème en question ; par exemple, 's'endormir' contrôle une seule position actancielle (' $\mathrm{X}$ s'endormir' = ' $\mathrm{X}$ commencer à dormir'), 'endormir' en contrôle deux ('Y endormir X' = 'Y causer que X s'endorme'), etc. Les actants sémantiques correspondent aux actants syntaxiques (grosso modo, les compléments essentiels) de la lexie qui exprime le sémantème en cause et font donc partie du schéma de régime (grosso modo, le cadre de sous-catégorisation) de cette lexie. Sur la théorie des actants dans le cadre Sens-Texte, voir Mel'čuk (2004). La seconde propriété des prédicats sémantiques est qu'ils dénotent des faits : actions ( $X$ construire $Y$; enquête sur $Y$ effectuée par $X$ ), événements ( $X$ naître; naissance de $X$ ), états (maladie de $X ;[X$ être]fatigué), propriétés (honnêteté de $X$ ), etc. Les prédicats types sont des verbes, des adjectifs et des adverbes, ainsi que des conjonctions et des prépositions, mais les prédicats peuvent aussi tout à fait correspondre à des noms.

- Les noms sémantiques sont des sens non liants dénotant des entités : ils ne contrôlent donc pas d'actants. L'exemple type d'un nom sémantique est le nom propre, mais il peut tout aussi bien s'agir d'un nom commun : sable, pierre, colline, etc.

- Les quasi-prédicats sémantiques représentent une classe intermédiaire de sens qui, tout en dénotant des entités - comme dans le cas des noms sémantiques -, contrôlent tout de même des actants - comme dans le cas des prédicats véritables. Les quasi-prédicats dénotent en fait des entités liées à des situations particulières, à des fonctionnements ou utilisations spécifiques de ces entités, etc., et c'est de ces faits sous-jacents qu'ils tirent leurs arguments. Par exemple, le quasi-prédicat VOISIN dénote une entité (un individu) liée dans une situation spécifique à une autre entité (un autre individu). Les quasi-prédicats s'expriment nécessairement par des noms.

L'ambivalence sémantique des NCL concerne des lexies qui, sémantiquement, appellent des actants tout en relevant à la fois de la sous-hiérarchie des étiquettes subordonnées à fait et de celle des étiquettes subordonnées à entité. En d'autres termes, si l'on se réfère aux définitions données ci-dessus, les NCL sémantiquement ambivalents expriment à la fois un prédicat et un quasi-prédicat sémantique. Des NCL comme QUESTION\#1a, INSULTE ou ELOGE\#2 [éloge de la démocratie] verront leur double nature, prédicative et quasi-prédicative, reflétée dans leurs propriétés de combinatoire - notamment, dans les types de collocatifs qu'ils contrôlent.

Après cette brève présentation de quelques notions théoriques et descriptives nécessaires à la caractérisation de l'ambivalence sémantique des NCL du français, nous pouvons passer à nos propositions pour résoudre les problèmes descriptifs qu'elle pose. Cela se fera en deux temps : présentation d'une hypothèse sur ce qui nous 
semble être la logique du phénomène de l'ambivalence sémantique des NCL et propositions pour un traitement lexicographique rigoureux des NCL ambivalents du français.

\section{Approche lexicographique du problème}

\subsection{Logique de l'ambivalence sémantique des NCL}

Pour étudier les NCL, nous avons examiné une centaine de noms décrits dans des ressources lexicales développées selon les principes de la Lexicologie Explicative et Combinatoire : le DiCo (précédemment mentionné), le Lexique actif du français (Mel'čuk \& Polguère, 2007), le Dictionnaire explicatif et combinatoire du français contemporain (Mel'čuk et al., 1984, 1988, 1992, 1999) et Dire autrement (Milićević \& Hamel, 2007). La plupart des NCL de notre corpus sont des dérivés sémantiques de verbes ; en termes de fonctions lexicales, il s'agit de $\mathbf{S}_{\mathbf{0}}$ (nom d'action), de $\mathbf{S}_{\mathbf{2}}$ (nom du second actant) ou de $\mathbf{S}_{\text {instr }}$ (nom d'instrument) de ces verbes. Si un NCL est ambivalent, il est lié au verbe dont il est le dérivé sémantique par une des deux paires de fonctions lexicales suivantes : $\mathbf{S}_{\mathbf{0}} / \mathbf{S}_{\mathbf{2}}$ ou $\mathbf{S}_{\mathbf{0}} / \mathbf{S}_{\text {instr. }}$. Par exemple :

- DISPUTE 'fait de se disputer' $\rightarrow \mathbf{S}_{\mathbf{0}}$ ( SE DISPUTER )

- CONSEIL\#I.1 'fait de conseiller et/ou ce qui est conseillé' $\rightarrow \mathbf{S}_{\mathbf{0}} / \mathbf{S}_{\mathbf{2}}$ ( CONSEILLER )

- CRItique\# . a 'fait de critiquer et/ou énoncé/texte par lequel on critique' $\rightarrow \mathbf{S}_{\mathbf{0}} / \mathbf{S}_{\text {instr }}$ ( CRITIQUER )

Dans nos données on trouve :

1. des NCL dénotant «purement» des actes de communication, ce qui est un cas de figure assez rare - par exemple, ENGUEULADE\#2 [Les caméras de télévision ont enregistré cette mémorable engueulade entre les membres du conseil d'administration.];

2. des NCL sémantiquement ambivalents, qui sont les plus nombreux et pour lesquels on note un débalancement dans la prédominance soit de la composante sémantique prédicative de communication langagière - par exemple, CONVERSATION\#a [écouter une conversation téléphonique] - soit de la composante quasi-prédicative (ce que, ce par quoi on communique) - par exemple, QUESTION\#1a [Vous ne répondez pas à la question.].

C'est bien entendu la seconde famille de NCL dont la description pose problème, notamment parce qu'il est nécessaire, pour assurer une description rigoureuse et cohérente de toutes les lexies de ce type, d'expliciter en vertu de quoi la plupart des francophones percevront que (i) CONVERSATION\#a dénote avant tout un acte et, secondairement, le «texte » correspondant, et que (ii) QUESTION\#1a dénote avant tout un énoncé et, secondairement, le fait de communiquer cet énoncé.

Avant de proposer une solution à ce problème, il est important de remarquer que notre corpus contient aussi des noms non ambivalents quasi-prédicatifs dénotant strictement les entités linguistiques impliquées dans la communication langagière :

- énoncés « purs »-par exemple, ENONCE\#1 [énoncé interrogatif ; énoncé de cinq mots], PHRASE\#1 [phrase claire, obscure, embrouillée], FORMULE\#I . a [les formules creuses des politiciens];

- textes « purs »-par exemple, ROMAN\#I.a [roman en deux volumes], THESE\#2 [thèse de doctorat], TORCHON\#II [Votre journal est un véritable torchon !].

On y trouve de même des noms (ambivalents ou non) dénotant des supports physiques de textes :

- documents - par exemple, INVITATION\#1b (= carton d'invitation\#1a) [Il a dû montrer son invitation à l'entrée.] ; 
- documents et textes correspondants - par exemple, TESTAMENT\#1 [désigner un héritier dans son testament], ORDONNANCE\#II [ordonnance du médecin].

De tels noms, puisqu'ils ne dénotent pas de façon première ou secondaire des communications langagières, n'entrent pas dans le paradigme des NCL. Ils sont cependant très intéressants pour nous dans la mesure où ils vont se rapprocher, de par leur sémantisme et, vraisemblablement, leur combinatoire, des NCL ambivalents qui sont de façon première quasi-prédicatifs (du type QUESTION\#1a).

Nous voudrions maintenant présenter une hypothèse sur ce qui nous semble être une certaine logique de la structuration sémantique des NCL ambivalents. Tout d'abord, il faut observer que, même s'il nous arrive de tergiverser lorsqu'il s'agit de diagnostiquer et décrire l'ambivalence sémantique des NCL, il est une opération que l'on peut effectuer de façon cohérente et «stable» (d'un lexicographe à l'autre) : il s'agit de la spécification du type de segment linguistique (la composante quasi-prédicative de l'ambivalence) dénoté par un NCL. Nous pouvons ainsi distinguer trois cas de figure, identifiés par les étiquettes sémantiques correspondantes :

1. énoncé - c'est-à-dire, segment linguistique caractérisé comme segment autonome, énonçable entre deux pauses longues;

2. texte - c'est-à-dire, ensemble structuré d'énoncés, tels que défini ci-dessus ;

3. qqch. que $l^{\prime}$ on dit (par écrit ou oralement) - c'est-à-dire, segment non spécifié en tant qu'énoncé ou texte.

Il est assez remarquable que l'on puisse prendre n'importe quel NCL sémantiquement ambivalent de notre corpus et dire, pratiquement sans hésitation, à quel cas de figure il correspond, pour ce qui est de la composante quasi-prédicative (segment linguistique) de son sens ; par exemple : QUESTION\#1a $\rightarrow$ énoncé, CONVERSATION\#a $\rightarrow$ texte, REPRIMANDE $\rightarrow$ qqch. que $1^{\prime}$ on dit.

Cette première observation nous a conduit à réaliser que l'étiquette sémantique énoncé était singulière, en contraste avec texte et qqch. que $l^{\prime}$ on dit, en ce qu'elle conférait un statut particulier aux lexies auxquelles elle s'appliquait : ces dernières se trouvent dénoter des véritables « artefacts » de communication et, dans le cas des NCL ambivalents, c'est cet aspect de leur sémantisme qui est prédominant. Cela nous amène à formuler une hypothèse qui tient en deux points :

\section{Hypothèse théorique et descriptive sur l'ambivalence sémantique des NCL du français}

A) tout NCL sémantiquement ambivalent qui dénote un énoncé dénote aussi l'acte de communication correspondant de façon secondaire ;

B) tout NCL sémantiquement ambivalent qui ne dénote pas un énoncé - qui dénote soit un texte soit reste vague sur ce plan (quelque chose que l'on dit) - dénote l'acte de communication de façon première.

En fonction de cette hypothèse, nous allons accepter comme valides trois patrons d'étiquettes sémantiques pour les NCL sémantiquement ambivalents, présentés ci-dessous par ordre croissant de prédicativité :

1. énoncé et/ou communication langagière-par exemple, QUESTION\#1a [Vous ne répondez pas à la question.], JURON\#1 [Il avance, les jurons à la bouche.] ;

2. communication langagière et/ou texte - par exemple, CONVERSATION\#a [écouter une conversation téléphonique], EXPOSE [Son exposé était long et ennuyeux.], TEMOIGNAGE\#I.2 [Les journalistes ont recueilli le témoignage d'une mère de famille.] ;

3. communication langagière et/ou qqch. que l'on dit - par exemple, OBJECTION\#1 
Nous parlons ici de patrons d'étiquettes dans la mesure où chacune des composantes énoncé, texte, qqch. que l'on dit et communication langagière pourrait être remplacée dans ces patrons par des étiquettes plus spécifiques de la hiérarchie.

L'identification et la normalisation de tels patrons présente un net avantage sur le flou qui règne maintenant dans la description des NCL sémantiquement ambivalents. En effet, ces patrons peuvent être utilisés de façon rigoureuse puisque, (i) une fois l'ambivalence identifiée, le choix entre énoncé, texte et qqch. que l'on dit comme composante quasi-prédicative semble s'établir de façon relativement consensuelle, et (ii) une fois cette composante quasi-prédicative identifiée, le choix du patron est automatique et univoque. Nous allons maintenant, en conclusion, présenter une stratégie de description de l'ambivalence sémantique fondée sur les observations qui viennent d'être faites à propos des NCL.

\subsection{Vers un traitement lexicographique rigoureux de l'ambivalence sémantique}

Pour conclure la présente étude, nous allons tenter de généraliser les observations qui ont été faites à propos des NCL pour proposer une stratégie lexicographique globale de traitement de l'ambivalence sémantique, stratégie dont nous illustrerons l'application en proposant les définitions pour quelques lexies françaises ambivalentes (voir l'Annexe, qui contient la définition lexicographique de six NCL ambivalents du français).

La stratégie que nous proposons pour traiter lexicographiquement les NCL repose sur quatre principes majeurs, qui seront présentés en allant du plus général (le moins lié au problème spécifique de l'ambivalence sémantique) au plus spécifique.

\section{A) Recours systématique à l'étiquetage sémantique}

Toute lexie étudiée doit se voir associer une étiquette sémantique, au sens où nous entendons ce terme, car les éventuelles généralisations que l'on fera sur la structuration sémantique de classes de lexies se formuleront en termes d'étiquettes sémantiques. Il est extrêmement important que l'étiquette sémantique ne soit pas conçue comme un simple «code» que l'on associe à la lexie, mais bien comme la composante centrale de sa définition, et il est nécessaire de développer (selon nous, de façon inductive), une hiérarchie d'étiquettes sémantiques rigoureusement testée sur le stock lexical de la langue (Polguère, 2003).

\section{B) Repérage méthodique de l'ambivalence sémantique}

Pour tester l'ambivalence sémantique, et notamment établir une distinction entre lexie sémantiquement ambivalente et acceptions distinctes d'un même vocable, il faut disposer d'une batterie de tests s'appuyant sur les caractéristiques de combinatoire des lexies en question - voir section 1.2 ci-dessus.

En effet, la bonne formulation de la définition lexicographique n'est pas un problème qui peut être traité les yeux rivés sur les sémantèmes eux-mêmes ou sur un niveau «conceptuel » d'interprétation de ceux-ci. Il s'agit surtout, en lexicographie, de rendre compte de la combinatoire des lexies définies, combinatoire qui nous guide dans la mise au point de la meilleure définition possible. Une fois l'ambivalence établie, celle-ci doit être formulée, comme nous l'avons indiqué en A) ci-dessus, en termes d'étiquettes sémantiques.

\section{C) Identification du caractère régulier/systématique de l'ambivalence}

Pour être bien formulée en termes d'étiquettes, l'ambivalence doit être caractérisée tout d'abord en fonction de son caractère régulier ou non. Tel qu'indiqué dans la section 2.1 , nous proposons de ne pas refléter directement dans la structure de l'étiquette sémantique une ambivalence régulière systématique, comme celle impliquée dans les «noms de professions» (individu qui exerce un métier, plutôt que individu qui exerce un métier ou le métier en question). 
C'est le positionnement d'une telle étiquette dans la hiérarchie (sous deux mères distinctes) et la règle de dérivation sémantique d'application systématique qui rendent compte de ce type d'ambivalence. Par contre, les ambivalences non systématiques, elles, doivent absolument être reflétées directement dans la structure de l'étiquette sémantique et, donc, dans une définition lexicographique bicéphale (possédant deux composantes centrales), car elles sont de nature beaucoup plus « lexicale ».

\section{D) Hiérarchisation des composantes sémantiques donnant lieu à l'ambivalence}

En cas d'ambivalence non systématique, qui doit donc être représentée dans la définition lexicographique au moyen de deux composantes centrales, il faut identifier quelle composante est prédominante. La méthode d'identification variera selon le type d'ambivalence traité. Ainsi, il n'est pas possible d'appliquer la stratégie utilisée dans la section 3.1 pour les NCL à une ambivalence comme celle impliquant les composantes 'animal' et 'espèce animale correspondante' mentionnée dans la section 1.2. Cette dernière ambivalence, fondée sur une disjonction exclusive, doit être décrite suivant une logique imposée par la règle de dérivation sémantique générale qui en est la source. Ainsi, en français, on constate l'implication suivante « nom (non terminologique) d'espèce animale $\Rightarrow$ nom d'animal » (cf. CHAT, VACHE, etc.), l'implication inverse n'étant pas vraie « nom d'animal $\nRightarrow$ nom (non terminologique) d'espèce animale » (cf. BICHE, TOREAU, etc.). Puisque la lexicalisation de 'espèce animale' n'existe pas sans celle de 'animal' (l'inverse n'étant pas vrai), on choisira comme étiquette générique en cas d'ambivalence animal ou espèce animale correspondante plutôt que espèce animale ou animal correspondant. (Insistons sur le fait que ce type de décision se fait directement en fonction des caractéristiques propres au lexique de la langue étudiée, et que les étiquetages sémantiques varieront nécessairement d'une langue à l'autre.)

Quels que soient les critères d'analyse utilisés, il faut absolument identifier une logique dans la structuration des étiquetages et, donc, des définitions, afin d'assurer la cohérence dans la description lexicographique. Cette logique sera fondée sur la combinatoire des lexies étudiées et sur la prédominance de tel ou tel type de lexicalisation dans la langue. La hiérarchisation des deux composantes sémantiques implique aussi le choix d'un connecteur approprié. Comme nous l'avons vu, il en existe deux : 1) la disjonction inclusive et/ou, lorsque la lexie ambivalente peut dénoter les deux composantes de façon simultanée (cf. XENOPHOBIE 'sentiment' et/ou 'attitude' ainsi, bien sûr, que les NCL examinés ici) ; 2) la disjonction exclusive ou, lorsque la lexie ambivalente ne peut dénoter simultanément les deux composantes (cf. CHAT 'animal' ou 'espèce animale').

Nous n'avons présenté que quatre principes lexicographiques pour le traitement de l'ambivalence sémantique, mais il est clair qu'il faudrait affiner la méthodologie que nous introduisons. De plus, nous sommes tout à fait conscients que l'hypothèse présentée dans la section 3.1 sur la logique de fonctionnement de l'ambivalence sémantique des NCL du français n'est, justement, qu'une hypothèse, qui demande qu'on la confronte beaucoup plus systématiquement que nous ne l'avons fait à la réalité des données lexicales. Nous pensons cependant avoir offert dans cette étude une stratégie opératoire, qui peut servir de guide pour une meilleure structuration des définitions lexicographiques. Finalement, il serait intéressant d'élargir le débat sur l'ambivalence sémantique en examinant, par exemple, la possibilité d'avoir dans la définition de lexies ambivalentes des composantes centrales qui seraient conjonctives, plutôt que disjonctives.

\section{Remerciements}

Nous tenons à offrir à Éva Buchi, Lidija Iordanskaja et Igor Mel'čuk, ainsi qu'à trois évaluateurs anonymes de CMLF'2010, nos remerciements les plus sincères (et sans ambivalence) pour leurs commentaires sur une version antérieure de ce texte. 


\section{Références bibliographiques}

Apresjan, Ju. (1974). Regular Polysemy. Linguistics 142, 5-32.

Barque, L. (2008). Description et formalisation de la polysémie régulière du français. Thèse de doctorat, Paris : Université Paris 7.

Barque, L. \& Polguère, A. (2009). Structuration et balisage sémantique des définitions du Trésor de la Langue Française informatisé (TLFi). In : Proceedings of the Fourth International Conference on Meaning-Text Theory (MTT'09), Montréal, 16-18 juin 2009.

Cruse, D. (1995). Polysemy and Related Phenomena from a Cognitive Linguistic Viewpoint. In : P. St-Dizier \& É. Viegas (dir.) : Computational Lexical Semantics. Cambridge (G.-B.) : Cambridge University Press, 33-49.

Dendien, J. \& Pierrel, J.-M. (2003). Le Trésor de la Langue Française informatisé : un exemple d'informatisation d'un dictionnaire de langue de référence. Traitement Automatique des Langues (t.a.1.), 44(2), 11-37.

Godard, D. \& Jayez, J. (1996). Types Nominaux et Anaphores ; le cas des objets et des événements. In : Anaphores temporelles et (in-)cohérence, Cahiers Chronos.

Jacquey, E. (2006a). Polysémie logique. In : D. Godard, L. Roussarie \& F. Corblin (dir.) : Sémanticlopédie : dictionnaire de sémantique. GDR Sémantique \& Modélisation, SNRS. [http://www.semantique-gdr.net/dico]

Jacquey, E. (2006b). Un cas de «polysémie logique »: modélisation de noms d'action en français ambigus entre processus et artefact. Traitement Automatique des Langues (t.a.1.) 47(1), 137-166.

Kleiber, G. (1999). Problèmes de sémantique : la polysémie en questions. Sens et structures. Villeneuve d'Ascq : Presses Universitaires du Septentrion.

Kleiber, G. (2008). Petit essai pour montrer que la polysémie n'est pas un sens interdit. In: J. Durand, B. Habert \& B. Laks (dir.) : Actes du Congrès mondial de linguistique française CMLF'08. Paris : Institut de linguistique française, 87-100. [http://dx.doi.org/10/1051/cmmlf08341]

Lexis (1992). Lexis. Dictionnaire de la langue française, Paris : Larousse.

Mel'čuk, I. (1996). Lexical Functions: A Tool for the Description of Lexical Relations in a Lexicon. In: L. Wanner (dir.) : Lexical Functions in Lexicography and Natural language Processing. Amsterdam/Philadelphia : Benjamins, 37-102.

Mel’čuk, I. (2001). Communicative Organization in Natural Language. Amsterdam/Philadelphia : Benjamins.

Mel’čuk, I. (2006). Explanatory-Combinatorial Dictionary. In: G. Sica (dir.): Open problems in linguistics and lexicography. Monza : Polimetrica, 225-355. [http://www.polimetrica.com/?p=productsList\&sWord=lexicography]

Mel'čuk, I. (2004). Actants in Semantics and Syntax I \& II. Linguistics 42(1 \& 2), 1-66 \& 247-291.

Mel'čuk, I. et al. (1984, 1988, 1992, 1999). Dictionnaire explicatif et combinatoire du français contemporain. Recherches lexico-sémantiques, vol. I-IV, Montréal : Les Presses de l’Université de Montréal.

Mel'čuk, I., Clas, A. \& Polguère, A. (1995). Introduction à la lexicologie explicative et combinatoire. Louvain-la-Neuve : Duculot.

Mel'čuk, I. \& Polguère, A. (2007). Lexique actif du français. Apprentissage du vocabulaire fondé sur 20000 dérivations sémantiques et collocations du français. Bruxelles : De Boeck \& Larcier.

Mel'čuk, I. \& Polguère, A. (2008). Prédicats et quasi-prédicats sémantiques dans une perspective lexicographique. Revue de linguistique et de didactique de langues LIDIL 37, 99-114.

Milićević, J. (1997). Étiquettes sémantiques dans un dictionnaire formalisé de type "Dictionnaire explicatif et combinatoire ». Mémoire de maîtrise, Montréal : Université de Montréal.

Milićević, J. \& Hamel, M.-J. (2007). Un dictionnaire de reformulation pour les apprenants du français langue seconde. In : G. Chevalier, K. Gauvin \& D. Merkle (dir.) : Les apports de la sociolinguistique et de la linguistique à l'enseignement des langues en contexte plurilingue et pluridialectal. Revue de l'université de Moncton, numéro hors série, 145-167. 
Nasr, A., Barque, L. \& Polguère, A. (2010). From the Definitions of the Trésor de la Langue Française to a Semantic Database of the French Language. In : Proceedings of EURALEX'2010, Leeuwarden (Pays-Bas), 6-10 juillet 2010.

Petit Robert (2010). Le nouveau Petit Robert, Paris : Dictionnaires Le Robert.

Polguère, A. (2000). Une base de données lexicales du français et ses applications possibles en didactique. Revue de linguistique et de didactique de langues LIDIL 21, 57-95.

Polguère, A. (2003). Étiquetage sémantique des lexies dans la base de données DiCo. Traitement automatique des langues (t.a.1.), vol. 44(2), 39-68.

Polguère, A. (2008). Lexicologie et sémantique lexicale. Notions fondamentales. Montréal : Les Presses de l'Université de Montréal.

Pustejovsky, J. (1995). The Generative Lexicon : A Theory of Computational Lexical Semantics. Cambridge (MA) : MIT Press.

Pustejovsky, J. (1998). The Semantics of Lexical Underspecification. Folia Linguistica 32, 324-347.

Wierzbicka, A. (1987). English Speech Act Verbs: A Semantic Dictionary. Sidney, etc. : Academic Press.

\section{Annexe \\ Exemples de définitions de NCL ambivalents du français}

Nous présentons des exemples de définitions de NCL ambivalents en les groupant selon les trois patrons d'étiquetage sémantique identifiés dans la section 3.1, sous Hypothèse théorique et descriptive sur l'ambivalence sémantique des NCL du français. Nous proposons deux définitions lexicales par patron.

Nos définitions ne sont pas de véritables définitions de la Lexicologie Explicative et Combinatoire : elles sont moins développées que ces dernières et se rapprochent plutôt des définitions que l'on trouve dans un dictionnaire commercial. Cette démarche nous semble justifiée dans la mesure où notre objectif principal est de proposer des définitions qui soient de bonnes solutions de remplacement pour les définitions de NCL sémantiquement ambivalents offertes dans les dictionnaires courants.

Pour chaque lexie décrite, nous fournissons les informations suivantes :

1. l'identification de la lexie accompagnée d'un ou plusieurs exemples ;

2. la forme propositionnelle, qui est une expression contenant la lexie et ses actants sémantiques, expression dont la définition est une paraphrase ;

3. la définition elle-même ;

4. des (quasi-)synonymes - fonction lexicale QSyn - et le verbe correspondant s'il existe - fonction lexicale $\mathbf{V}_{\mathbf{0}}$;

5. des commentaires éventuels.

\section{Premier patron : énoncé et/ou communication langagière}

\section{QUESTION\#1a}

Aude, la formatrice, a souvent entendu cette question au cours des stages qu'elle anime: "Par où je commence?». 
Question\#1a « $Y$ » posée par $X$ à $Z$ au sujet de $W$

'Énoncé « $\mathrm{Y}$ » par lequel l'individu $\mathrm{X}$ demande à l'individu $\mathrm{Z}$ de l'information au sujet de $\mathrm{W}$ et/ou énonciation de $\mathrm{Y}^{\prime}$

- QSyn : interrogation, demande

- $\mathbf{V}_{\mathbf{0}}:$ demander

\section{JURON\#1}

Il se démène dans la foule, bâton à la main et jurons aux lèvres.

L'étranger lança un juron bref, mais énergique.

Qui n'a jamais lancé un juron en se coinçant le doigt ou en se cognant le coude?

Juron\#1 « $Y$ » émis par Xà cause de $\mathrm{Z}$

$=$

'Exclamation « $\mathrm{Y} »$ familière ou grossière par laquelle l'individu $\mathrm{X}$ manifeste une colère et/ou surprise provoquée par le fait $\mathrm{Z}$

et/ou

énonciation de $\mathrm{Y}^{\prime}$

- QSyn : blasphème; gros mot; exclamation ; vieilli jurement

- $\mathbf{V}_{\mathbf{0}}$ : jurer

Commentaires :

1. Formellement, il serait possible de faire ici l'économie de la composante disjonctive 'et/ou énonciation de Y', en laissant à la composante centrale 'exclamation', elle-même sémantiquement ambivalente, le soin de modéliser l'ambivalence sémantique de JURON\#1. Il nous semble cependant préférable de ne pas utiliser ce type de modélisation implicite par récursion dans le cas d'une propriété aussi importante pour la combinatoire sémantique que l'ambivalence. Cette remarque vaut pour toutes les définitions de NCL qui suivent.

2. Le verbe correspondant JURER\#II est défini à partir du nom JURON\#1, et non l'inverse : $X$ jure\#II « $\mathrm{Y}$ » à cause de $\mathrm{Z}=$ ' $\mathrm{X}$ énonce le juron\#1 « $\mathrm{Y}$ » à cause de $\mathrm{Z}$ '.

\section{Deuxième patron : communication langagière et/ou texte}

\section{EXPOSE}

Plus de deux cents personnes ont écouté son exposé sur les stratégies de communication.

Exposé de $X$ sur $Y$ à $Z$

$=$

'Présentation orale faite par l'individu $X$, par laquelle $X$ communique au groupe d'individus $Z$ un ensemble structuré d'informations et/ou d'idées à propos de $\mathrm{Z}$

et/ou

le texte correspondant'

- QSyn : compte rendu, fam topo ; communication, conférence, discours ; fam laïus 


\section{Commentaires :}

1. Le vocable est en réalité polysémique, mais le DiCo, notre base lexicographique de référence, ne contient la description que d'une acception (exposé oral). En théorie, la lexie analysée ici devrait porter un numéro distinctif l'identifiant précisément en tant qu'acception du vocable EXPOSE.

2. Il n'y a pas de verbe correspondant : EXPOSE n'est pas une nominalisation de EXPOSER [qqch. à qqn.].

\section{TEMOIGNAGE\# I. 2}

Les journalistes ont recueilli le témoignage d'une mère de famille, confrontée à l'impossibilité de trouver un logement.

Témoignage\#I . 2 de X au sujet $Y$ auprès de $Z$

'Communication langagière par laquelle l'individu $\mathrm{X}$ témoigne\#I.2 au sujet du fait $\mathrm{Y}$ auprès de l'individu Z

et/ou

le texte correspondant'

- $\quad$ QSyn : version des faits; récit; histoire ; rapport

- $\mathbf{V}_{\mathbf{0}}$ : témoigner

\section{Commentaires :}

1. Rappelons que texte $=$ ensemble d'énoncés.

2. La définition approximative $\mathrm{du}$ verbe TEMOIGNER\#I.2 est : $\mathrm{X}$ témoigne\# I. 2 au sujet de $\mathrm{Y}$ auprès de $\mathrm{Z}$

'L'individu $\mathrm{X}$ communique à l'individu $\mathrm{Z}$ les informations concernant le fait $\mathrm{Y}$ telles qu'elles proviennent de l'expérience personnelle de $\mathrm{X}$ et que $\mathrm{X}$ croit conformes à la réalité dans le but que soient établis les faits relatifs à $\mathrm{Y}^{\prime}$.

\section{Troisième patron : communication langagière et/ou ce qu'on dit}

\section{OBJECTION\#1}

Si le département compétent adresse une objection aux cantons contractants, la première étape consiste à rechercher une solution à l'amiable.

Jung émit de fortes objections à une telle conception au motif que dénier au mal une réalité serait revenu à en ignorer la signification.

Objection\# 1 de $X$ consistant en $Y$ adressé à $Z$ relativement à $W(Z)$

'Affirmation par la personne $\mathrm{X}$ du fait $\mathrm{Y}$ à la personne $\mathrm{Z}$ en réaction à l'affirmation $\mathrm{W}$ de $\mathrm{Z}$ avec laquelle $\mathrm{X}$ n'est pas d'accord 
et/ou

les propos correspondants'

- QSyn : réponse, réplique, contradiction, réfutation

- $\mathbf{V}_{\mathbf{0}}$ : objecter\#1

\section{Commentaire :}

La composante 'personne', qui effectue ci-dessus un typage sémantique des variables actancielles ' $\mathrm{X}$ ' et ' $\mathrm{Z}$ ', est bien distincte de 'individu' : elle dénote un individu et/ou une entité sociale regroupant un ensemble d'individus (syndicat, gouvernement, etc.); cf. les expressions personne physique (individu) vs personne morale (entité sociale).

\section{CRITIQUE\# I . a}

Si votre interlocuteur vous lance une critique qui vous déplaît, répondez par un argument qui coupe court à toute discussion.

En 1942, il publie un nouveau livre "Introduction à la politique économique », où il exprime sa vive critique du dirigisme.

Critique\#I . a de X envers $Y$ au sujet de $Z(X)$

'communication langagière par laquelle la personne $\mathrm{X}$ critique\# I. a la personne $\mathrm{Y}$ pour le fait $\mathrm{Z}$ et/ou

les propos correspondants'

- QSyn : attaque, blâme\#1, désapprobation, reproche\#1b, reproche\#2

- $\quad \mathbf{V}_{\mathbf{0}}$ : critiquer\#I. a

\section{Commentaire :}

Le quatrième volume du Dictionnaire explicatif et combinatoire du français contemporain (Mel'čuk et al., 1999) offre la définition suivante pour CRITIQUER\# I . a : X critique\# I . a Y pour $Z={ }^{\prime} \mathrm{X}$ exprime une opinion défavorable sur la personne $\mathrm{Y}$ au sujet du fait $\mathrm{Z}$ dont $\mathrm{X}$ tient $\mathrm{Y}$ responsable - certains aspects de $Z$ étant perçus par $X$ comme mauvais pour $X$ ou ses semblables - dans le but que $\mathrm{Y}$ et/ou ses semblables bénéficie(nt) de l'opinion de $\mathrm{X}$ et que $\mathrm{Z}$ soit rectifié. Cette définition est bien entendu d'une structure très complexe et il serait souhaitable d'en produire une version " allégée » de type dictionnaire grand public, dans la lignée des définitions de NCL proposées ici. 